\title{
An Investigative Study on the Listening Comprehension Strategies Employed in International English Language Testing System (IELTS)
}

\author{
Shang-chun Zeng \\ College of International Exchange \\ Wuzhou University \\ Wuzhou, Guangxi, CHINA \\ 123209352@qq.com
}

\author{
Ying Shen* \\ Foreign Languages College \\ Guangxi University \\ Nanning, Guangxi, CHINA \\ *ying_kuomei@aliyun.com
}

\begin{abstract}
This study aims to explore the current situation of using Listening Comprehension Strategies (LCS) in IELTS, compare the difference in using LCS between the efficient listeners and less efficient listeners, find out efficient learners' specific methods of applying LCS, and provide some feasible suggestions for developing these strategies. This study collected IELTS listening scores of 166 sophomores from Sino-Canadian International College (SCIC) in November, 2015 and then investigated their uses of LCS through questionnaire. The result revealed that: First, students were able to use five LCS to a certain degree, and memory strategy was the most popularly used while meta-cognitive strategy was the least popularly used. Second, there was a significant positive relationship between LCS and IELTS listening achievements. Third, a significant difference was found between efficient listeners and less efficient listeners in using LCS. Fourth, no significant difference between male and female students appeared in LCS usage. Fifth, top students especially emphasized inferring, association, clause comprehension, sound recognition, and self-monitoring.
\end{abstract}

Keywords_IELTS Listening; Listening Comprehension Strategies; Listening Teaching; Efficient Listeners

\section{INTRODUCTION}

For a long time, listening comprehension has been neglected in IELTS listening training course, where teachers focus more on the score result than on the listening process during which students are supposed to apply various LCS. In the IELTS listening class, the teacher usually follows "the more the better principle" in terms of the quantity of listening material, as such students easily get bored or unmotivated when playing the role of so-called "tape-recorder". It is true that there is no separate listening course for students to Fig. out and train their LCS. However, it should be noted that LCS is generally regarded as one of the significant components of learning strategies. According to Vandergrift's (1996) [3] study, LCS can be divided into three major categories: Meta-cognitive, cognitive and social/affective strategies. Oxford's (1990) [5] LCS is also very influential and more comprehensive because she makes a general distinction between direct and indirect LCS. Direct LCS includes memory strategies such as association, applying images and sounds, cognitive strategies such as analyzing and reasoning and creating structure for input and output, compensation such as predicting, referencing and guessing intelligently; Indirect LCS consists of meta-cognitive strategies such as making learning plan and self-evaluating, affective strategies such as self-encouraging, and social strategies such as cooperating and communicating with others.

\section{ReSEARCH METHODOLOGY}

\section{A. Research Subjects}

The participants in this research were 166 second year students in 4 intact classes in SCIC, Guangxi University, including 102 females (61\%) and 64 males (39\%). Because of the test system and curriculum design in SCIC, all of them have to take IELTS test twice a semester as the mid-term and final exams, and also they have received regular IELTS training. Thus, they all have ILETS listening experience. The following bar chart (see Fig. 1) gives us a clear picture of the participants' scores in recent IELTS listening test taken in November.

\section{B. Research Questions}

Research questions in this study are mainly as follows:

(1) What is the overall situation of LCS used by IELTS test takers?

(2) Is there any correlation between LCS use and IELTS listening achievements?

(3) What are the distinctive features in LCS used between male and female IELTS test takers?

(4) What are the distinctive features in LCS between efficient and less efficient IELTS test takers in IELTS listening?

(5) What are the main features of LCS employed by top IELTS test takers in IELTS listening? 


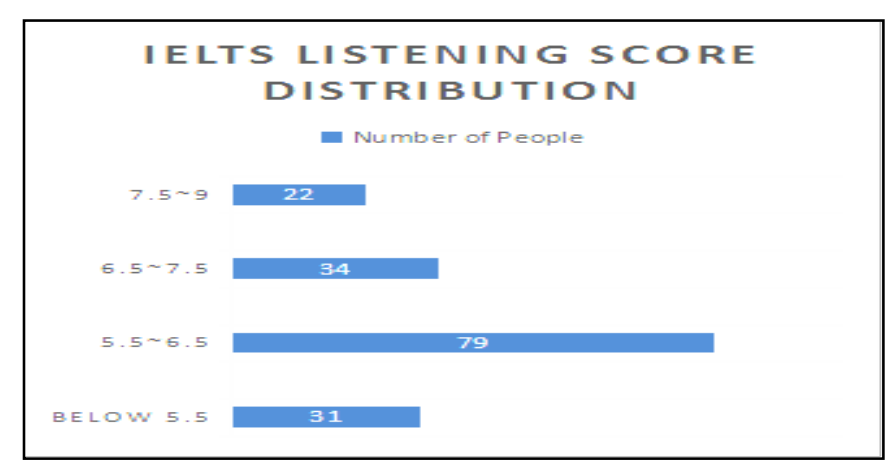

Fig. 1. IELT Listening Score’s Distribution

\section{Research Methods}

\section{1) Questionnaire}

We used a self-designed LCS questionnaire, which is one of the essential instruments based on the framework of Oxford's (1990) [5], and O’Malley and Chamot's (1989) [1] classifications about LCS.

The questionnaire was divided into two parts. The first part was mainly about personal information, and there were 4 questions covering gender, average score range, correspondents' personal opinions about the status of IELTS listening and the most important factor affecting IELTS listening. The second part composed of 32 items of listening comprehension behaviors or activities, each of them represented a specific set of strategy items: (a) Meta-cognitive strategies (Items 1 to 8), (b) Cognitive strategies (Items 9 to 15), (c) Memory strategies (Items 16 to 21), (d) Compensation strategies (Items 22 to 27), and (e) Social/affective strategies (Items 28 to 32). The respondents assigned a value to each item on a five-point Likert scale.

\section{2) Interviews}

Supplementary interviews were carried out in order to collect more information for this study. We chose the top 10 students including 3 boys and 7 girls from the 166 subjects to have a face-to-face interview. The interviews were conducted in Chinese so that interviewees can express their ideas more smoothly. Five questions were included in the interviews as follows.

(1) From your point of view, what is the key competence for IELTS listening?

(2) What methods do you usually use to help and improve your listening comprehension? Please explain more specifically.

(3) Will you subconsciously use some methods to help you comprehend during the IELTS listening?

(4) What methods will you apply to help you collect and memorize the useful information during the IELTS listening? Please explain more specifically.

(5) What methods will you apply to help listening comprehension when the whole IELTS listening test is finished? Please explain more specifically.

\section{RESULTS}

\section{A. Overall Pattern of LCS}

The questionnaire responses were recorded and entered into SPSS 19.0. Descriptive statistics is shown in Table 1.

TABLE I. DESCRIPTIVE Statistics OF THE OVERALL STRATEGy USE

\begin{tabular}{llllll}
\hline & $\mathrm{N}$ & Minimum & Maximum & Mean & Std. Deviation \\
\hline Meta-cognitive strategies & 166 & 1.38 & 5.00 & 3.2048 & .65252 \\
Cognitive strategies & 166 & 1.71 & 5.00 & 3.4036 & .69772 \\
Memory strategies & 166 & 1.83 & 5.00 & 3.6456 & .70069 \\
Compensation strategies & 166 & 1.00 & 5.00 & 3.2068 & .56681 \\
Social/Affective strategies & 166 & 1.60 & 5.00 & 3.4000 & .69805 \\
Valid N (listwise) & 166 & & & &
\end{tabular}

According to Oxford's Strategy Inventory for Language Learning (SILL), each mean score indicates the strategy's frequency of use: 1.0-1.4 refer to "Never Use This Strategy"; 1.5-2.4 mean "Seldom Use This Strategy"; 2.5-3.4 refer to "Sometime Use This Strategy"; 3.5-4.4 mean "Often Use This Strategy"; 4.5-5.0 refer to "Always Use This Strategy". In other words, a strategy with a mean score above 3.0 could be regarded as a frequently used strategy. From Table 1, it is easy to see that the five strategies are all used frequently by the IELTS listeners, which indicates that those listeners have realized and are able to use certain LCS. This result also supports Bacon's (1992) [6] statement that listening comprehension is characterized by active processing and by the use of LCS. Among the five strategies, memory strategies are the most popular with a mean score 3.6456, which manifests that students are used to associating while listening. Cognitive strategies (mean score 3.4036) are also thought highly of by IELTS listening test takers, compared with social/affective strategies (mean score 3.4). Compensation strategies (mean score 3.2068) are considered sometimes used by them. In addition, it is surprising to find that meta-cognitive strategies rank the bottom with only 3.2048, which implies that most of the listening test takers are not good at planning, selfevaluating. 


\section{B. Relationship between LCS and IELTS Listening Achievements}

Pearson correlation coefficient (r) between meta-cognitive strategies, cognitive strategies, memory strategies, compensation strategies, social/affective strategies and IELTS listening achievement are 0.319, 0.335, 0.258,0.166, 0.276 receptively, this indicates that there is a positive correlation between the five strategies and the IELTS listening achievements to some extent.

\section{Comparison between High-scored Listeners and Low- scored Listeners}

The number of high-scored listeners (IELTS listening score is $\geqslant 6.5$ ) and low-scored listeners (IELTS listening score is $\leqslant$ 6.5) are 56 and 110 respectively (see Fig 1). The independentsamples t-test is used to explore the differences in LCS use between these two groups.

According to the Sig. value we can see that there is a significant distinction in LCS use between high-scored and low-scored listeners. Listeners with a higher IELTS listening score are more inclined to use those strategies, while listeners with a relatively lower score are lack of the ability to use them. More concretely, in terms of the strategies used by high-scored listeners, memory strategies (mean score 3.84) rank the first as they prefer to associate, recall memory, organize memory for comprehension. The second rank for high-scored listeners is the cognitive strategies (mean score 3.70), such as building vocabulary, note-taking, and knowledge transferring. These are mainly taught in class. It is interesting to notice that social/affective strategies and meta-cognitive strategies have a very close mean score at being around 3.4 , which implies that good listeners of IELTS are capable of cooperating, lowering anxiety, forming self-confidence, doing self-management and self-evaluation. Compensation strategies (mean score 3.34) like inferring, guessing, and avoiding listening barriers ranking the bottom are probably due to the reason that listeners are still not equipped with enough background knowledge base. The most significant difference lying in low-scored listeners and highscored ones is in the use of cognitive strategies although they are typically found to be the most popular strategies used by language learners in the past.

\section{Comparison between Male and Female Listeners}

The different use of LCS between male and female listeners taking the IELTS listening test is made clear by means of Pearson correlation test. The majority of listeners are females, occupying nearly 61\%, while male listeners account for only $39 \%$ of the total amount. It is obvious that there is no notable difference in the use of the five LCS between genders (all $\mathrm{p}>0.05)$. Very surprisingly, in the five strategies, memory strategies are employed with a higher mean by males (3.7042) than by females (3.6088). However, the rest of the strategies including meta-cognitive strategies, cognitive strategies, compensation strategies and social/affective strategies are more frequently used by female listeners.

\section{E. Results from the Interviewees}

Top 10 students ( 7 girls and 3 boys) with the highest IELTS listening score are selected to have the interview. Some typical thoughts and ideas about LCS from these students can be summarized as below.

First, as for the first question about the key competence for IELTS listening, half of the students gave the answer that speech recognition and clauses comprehension are the most crucial factors. Five students regarded self-planning or selfmonitoring before the formal IELTS listening test as the key. Because speech recognition and clauses comprehension both belong to cognitive strategies, and advance planning as well as self-monitoring are classified into meta-cognitive strategies, these two LCS received more attention of not only intermediate level students but also IELTS listening teachers.

Second, as to the second question about the preparation before IELTS listening begins, almost everyone mentioned using the vocabulary of testing point and given topics as well as given question types to predicate answers, which is a typical behavior of using cognitive strategies. In addition, lowering anxiety and concentration were also mentioned, which belongs to social/affective strategies.

Third, considering the third question, only 4 students said they applied certain LCS subconsciously. In other words, despite having a high IELTS listening score, they were still not able to use appropriate LCS subconsciously.

Fourth, with regard to the fourth question concerning the use of LCS while listening, almost every student said that they used inferring strategy based on context clues, catching up key words, giving selective attention which are classified into compensation strategies. Eight of them mentioned that they used scene association, background knowledge transferring that are parts of memory strategies. Six of them said that they used note-taking, translation, and elaboration, but sometimes they failed to use them when they tried to catch up with the speech speed.

Fifth, in relation with the last question about what they usually do when the listening test ends, most of them answered that they would double check the answers on the answer sheet or predicate their scores according to their own feeling.

\section{CONCLUSIONS}

The overall pattern of LCS used by IELTS listeners is in agreement with the study results concluded by O'Malley et al. [2] as that "listening comprehension is an active process in which the listeners construct meaning by using strategic resources to fulfill the task requirement”. The participants of this study employed all the five LCS in which cognitive strategies and memory strategies were used most frequently, however, compensation strategies, meta-cognitive strategies and social/affective strategies did not enjoy a high popularity.

IELTS listening achievements were influenced by LCS based on a positive relationship being found between LCS and listening achievements. Judged by the correlation coefficient, cognitive strategies had the closest relationship with IELTS listening achievements, and then meta-cognitive strategies, 
memory strategies, social/affective strategies, and compensation strategies. It should be especially noted that meta-cognitive strategies also had a relatively higher correlation coefficient, and this result also proved Vandergrift's (1998) [4] findings that meta-cognitive strategies have positive effect on listening proficiency because they help the listeners become active participants in their listening performance.

A statistically significant difference existed between highscored and low-scored listeners in the use of LCS of IELTS listening testing. Students with a higher IELTS listening rank used meta-cognitive strategies and cognitive strategies more often, whereas low-scored students depended much on social/affective strategies. Both of the groups applied memory strategies at a very high frequency.

There was no statistically significant difference between male and female students on the use of LCS in IELTS listening. Male students were found to employ memory strategies more often than female students; however, female students used the other four strategies more frequently than male students.

Fifth, top students with an average IELTS listening rank above 8 attached great importance to using cognitive strategies, compensation strategies and memory strategies. They were also found to think highly of meta-cognitive strategies. However, they are not used to employing social/affective strategies very often.

\section{SugGestions}

It has been clearly proven that IELTS listeners have more or less employed the five LCS to assist them in comprehending the listening test in this study. However, there is still a need for them to deepen the understanding of LCS and have a better idea of using them appropriately. Thus, some pedagogical suggestions are put forward as below.

First, teachers need to raise students' awareness in using LCS subconsciously. Using LCS consciously in the IELTS listening test is better than using them unconsciously.

Second, enhance students' ability of self-monitoring and inference. These two abilities belong to meta-cognitive strategies and compensation strategies respectively, which are used less frequently as indicated in the major findings. Teachers should help students understand the close relationship between meta-cognitive strategies and cognitive strategies because the function of cognitive strategies is greatly influenced by meta-cognitive strategies.

Third, focus on the teaching of relevant culture background knowledge. River (1983) [7] said, "Language cannot be separated from its culture, because it is deeply rooted in culture.”

Fourth, combine IELTS listening skills with other three skills. Language skills are made up of listening, speaking, reading and writing. Although listening is the most basic skill, other skills also have a very strong backwash on listening.

\section{ACKNOWLEDGMENTS}

This research is financially supported by Innovative Research Foundation for National University Students in 2014 (NO1410593049).

\section{REFERENCES}

[1] J. O'Malley \& Chamot, A., Listening Comprehension Strategies in Second Language Acquisition. Applied Linguistics, 10 (4), 1989: 428436.

[2] J. O’Malley, Chamot, A., \& Kupper, L, Learning Strategies in Second Language Acquisition. Shanghai: Shanghai Foreign Language Education Press, 2001.

[3] L. Vandergrift, The listening comprehension strategies of core French school students. The Canadian Modern Language Review, 52 (2), 1996: 200-223.

[4] L. Vandergrift, Successful and less successful listeners in French: what are the strategy differences? The French Review, 71(3), 1998: 370-390.

[5] R. L. Oxford, Language Learning Strategies: What Every Teacher Should Know. Boston: Newbury House. 1990.

[6] S. Bacon, The relationship between gender, comprehension, processing strategies, and cognitive and affective response in foreign language listening. The Modern Language Journal, 76, 1992: 160-162.

[7] W.M. River, Teaching of Translation. Chicago and London: The university of Chicago Press. 1983. 